\title{
Pontes de miocárdio em bovinos azebuados
}

\author{
Miocardial bridges in zebu bovines
}

\author{
Renato Souto SEVERINO'; Frederico Ozanan CARNEIRO e SILVA'; André Luiz Quagliatto \\ SANTOS'; Sérgio Salazar DRUMMOND'; Pedro Primo BOMBONATO²; Fernando Proni \\ DURAN'; Amilton Vallandro MARÇAL ${ }^{3}$
}

CORRESPONDENCE TO Pedro Primo Bombonato Departamento de Cirurgia Faculdade de Medicina Veterinária de Medicina da USP Veterinaria e Zootecnia da USP Av. Prof. Dr. Orlando Marques de Paiva. 87 - Cidade Universitária Armando de Salles Oliveira 05508-000 - São Paulo - SP. Brasil

e-mail: bombonat@usp.br

1 - Universidade Federal de Uberlândia, $M G$

2 - Departamento de Cirurgia Faculdade de Medicina Veterinária e Zootecnia da USP 3 - Pontifícia Universidade Católica, RS

\section{RESUMO}

Estudou-se em 30 corações de bovinos azebuados, machos, com idade variando de 30 a 50 meses, a freqüência, a largura, a posição e a vasculotopia das pontes de miocárdio, mediante injeção das artérias coronárias com solução de Neoprene látex 450 e posterior dissecação. Os resultados indicam freqüência de $100 \%$ de corações com pontes e $56,6 \%$ das peças apresentaram pontes múltiplas. A largura das pontes variou de $0,3 \mathrm{~cm}$ a $6,2 \mathrm{~cm}$ com média de $1,62 \mathrm{~cm}$ e estas localizaramse com maior freqüência na região média $(36,8 \%)$ do ventrículo. O ramo interventricular paraconal foi o que apresentou maior número e freqüência de pontes.

UNITERMOS: Bovinos; Pontes de miocárdio; Coração; Artérias.

\section{INTRODUÇÃO}

$\mathrm{P}$ erante o crescente avanço da angiologia, em especial da cardiologia, nas áreas aplicadas, faz-se necessário oferecer, paralelamente, conhecimentos morfológicos, especialmente nas áreas onde os estudos são mais parcimoniosos, principalmente tratando-se de animais domésticos, conforme nos certifica a literatura consultadia. Ademais, alicerçados neste contexto e até mesmo com a expectativa futura de que se possa estabelecer o perfil global sobre a presença de pontes de miocárdio nos diferentes animais domésticos e suas interações com processos patológicos, como os já atribuídos ao homem, optamos por estudar as pontes de miocárdio em bovinos azebuados, pelo fato de serem enquadrados como autênticos representantes da pecuária nacional. já que são criados em grande escala no Brasil. Oriundos do cruzamento de Bos indicus, cuja aptidão é o fornecimento de carne, com Bos taurus, de aptidão para leite, esse grupo de animais tem mostrado peculiaridades morfológicas distintas daquelas originalmente presentes nas espécies citadas. Assim sendo, procuramos estudar neste trabalho a ocorrência de pontes de miocárdio sobre os ramos das artérias coronárias em corações de bovinos azebuados e avaliá-las sistematicamente no que tange à frequiência, largura e localização das mesmas.

\section{MATERIAL E MÉTODO}

Utilizamos nesta pesquisa 30 corações de bovinos azebuados, adultos, machos, com idade variando de 30 a 50 meses, abatidos em frigoríficos das regiōes do Triângulo Mineiro (MG) e de Campinas (SP).

Após a remoção dos órgãos sem patologias cardíacas aparentes, esvaziamos suas cavidades, mediante massagens manuais, e as lavamos em água corrente, eliminando assim a formação de possíveis coágulos no seu interior.
O preparo das peças fazia-se mediante canulação dos óstios coronarianos e lavagem com solução fisiológica de todo o trato para, em seguida, preenchermos esses sistemas com solução de Neoprene látex 450, corado com pigmento específico (Du Pont do Brasil S/A).

Posteriormente, fixamos as peças em uma solução aquosa de formol a $10 \%$, durante um período de no mínimo 48 horas, para a seguir dissecarmos as artérias coronárias direita e esquerda com seus respectivos ramos ventriculares, com auxílio de instrumentos cirúrgicos adequados, valendo-nos, sempre que necessário, do campo visual de uma lupa monocular Wild $(10 \mathrm{x})$, salvaguardando sempre o comportamento das fibras do miocárdio.

Após a identificação das pontes de miocárdio, sua localização foi anotada e suas larguras foram mensuradas com auxílio de paquímetro. O mesmo procedimento foi adotado em relação às respectivas alturas do ventrículo, sempre tomadas na face esquerda a partir da margem dorsal do sulco coronário até o ápice cardíaco.

Para a descrição dos resultados adotamos nomenclatura anatômica utilizada por Hegazi ${ }^{15}$ (1958) com os reparos de Habermehl ${ }^{13}$ (1959), no que se refere à nominação das artérias coronárias e seus ramos ventriculares. Ainda, submetemos os resultados a tratamento estatístico executado com auxílio do software "Statgraphics - Statistical Graphics System" - versão 2.6, do qual nos valemos de sumários estatísticos, teste de correlação de Pearson com nível de significância de $1 \%$, método Kruskal - Wallis com alfa $=0,05$.

\section{RESULTADOS}

Após a dissecação do sistema arterial coronariano, pudemos observar:

\section{Freqüência}

Todos os 30 corações estudados apresentavam pontes de 
miocárdio em ramos das artérias coronárias, que perfaziam o total de 106 pontes, das quais $100(94,3 \%)$ estavam localizadas sobre ramos da artéria coronária esquerda e $6(5,6 \%)$ sobre ramos da artéria coronária direita.

Foram identificadas de 1 a 7 pontes de miocárdio por coração, distribuídas da seguinte maneira : com I ponte $4(13,3 \%$ ) corações; com 2 pontes $5(16,6 \%)$ corações; com 3 pontes $6(20,0 \%)$ corações; com 4 pontes $6(20,0 \%)$ corações; com 5 pontes $5(16,6 \%)$; com 6 pontes $3(10,0 \%)$ corações e com 7 pontes $1(3,3 \%)$ coração.

\section{I.argura}

Observamos que a largura das 106 pontes de miocárdio encontradas variava de $0,3 \mathrm{~cm}$ a $6,2 \mathrm{~cm}$, com média de $1,62 \mathrm{~cm}$, em corações cuja altura ventricular estava comprendida entre $14,9 \mathrm{~cm}$ e $20,0 \mathrm{~cm}$, com média de $16,9 \mathrm{~cm}$.

No referente à largura das pontes em relação a sua presença nas artérias coronárias, verificamos que a de $94.3 \%$ das pontes de miocárdio identilicadas sobre ramos da artéria coronária esquerda variava de $0,3 \mathrm{~cm}$ a $6,2 \mathrm{~cm}$, com média de $1,62 \mathrm{~cm}$. Já as sobrepostas à artéria coronária direita mostravam larguras compreendidas entre $0.6 \mathrm{~cm}$ e $3,0 \mathrm{~cm}$, com média de $1,71 \mathrm{~cm}$.

\section{Localização}

\section{Posição}

Para a descrição da posição das pontes de miocárdio, dividimos os ventrículos eqüitativamente em 4 regiões, assim designadas: dorsal, média, ventral e apical, e esta última teve como ponto de reparo dorsal a curvatura do sulco interventricular paraconal da face esquerda para a face direita e como ponto ventral o ápice cardíaco. As pontes de miocárdio por nós identificadas localizavam-se com maior freqüência: na regiâo média $39(36,8 \%)$ pontes, na região apical $26(24,5 \%)$ pontes, na região ventral 15 $(14.1 \%)$ pontes, na região dorsal $12(11.3 \%)$ pontes, na região dorsal e média conjuntamente $10(9,4 \%)$ pontes e nas regiões média e ventral $4(3,7 \%)$ pontes. De $94,3 \%$ das pontes de miocárdio observadas em ramos da artéria coronária esquerda, $37(35.0 \%)$ pontes encontravam-se instaladas na região média, $26(24,5 \%)$ na região apical, $15(14,1 \%)$ na região ventral, $10(9.4 \%)$ nas regiões dorsal e média concomitantemente, $8(7,5 \%)$ na região dorsal e 4 $(3,7 \%)$ nas regiões média e ventral, enquanto, das $6(5,6 \%)$ pontes locadas em ramos da artéria coronária direita, $4(3,7 \%)$ encontravamse na região dorsal e $2(1,8 \%)$ na região média.

\section{Vasculotopia}

Das pontes de miocárdio presentes em ramos da artéria coronária esquerda $(94,3 \%)$ notamos, mais freqüentemente, no ramo interventricular paraconal $42(39,6 \%)$ pontes, seguidas daquelas sobre o ramo colateral proximal - $15(14,1 \%)$ pontes, ramo colateral distal - $14(13,2 \%)$ pontes, ramo proximal ventricular esquerdo- 12 $(11.3 \%)$ pontes, ramo interventricular subsinuoso $-9(8,5 \%)$ pontes e ramo distal ventricular esquerdo $-8(7,5 \%)$ pontes. Nas $6(5,6 \%)$ pontes de miocárdio restantes observadas em colaterais da artéria coronária direita, visibilizamos no ramo circunflexo direito $-4(3,7 \%)$ pontes e ramo proximal ventricular direito $-2(1,9 \%)$ pontes.

\section{Pontes múltiplas}

Em 17 corações $(56,6 \%)$ notamos a ocorrência de pontes de miocárdio múltiplas, que variavam de 2 a 4, sempre sobre o mesmo vaso. Assim, identificamos com maior freqüência pontes múltiplas no ramo interventricular paraconal $4(3,7 \%)$ pontes, 1 vez, $3(2,8 \%)$ pontes 3 vezes e $2(1,9 \%)$ pontes 6 vezes; no ramo colateral distal $3(2,8 \%)$ pontes I vez e $2(1,9 \%)$ pontes 2 vezes, no ramo interventricular subsinuoso $3(2,8 \%)$ pontes I vez e 2 (1,9\%) pontes 1 vez; no ramo circunflexo direito $2(1,9 \%)$ pontes I vez e no ramo proximal ventricular esquerdo $2(1,9 \%)$ pontes I vez.

\section{Aspecto da análise estatística}

No que se refere aos valores mensurados relativos à altura ventricular dos corações que apresentavam pontes de miocárdio, pudemos anotar como mediana, moda, variância, desvio padrão, erro padrão, respectivamente: $17,1 \mathrm{~cm} ; 17,5 \mathrm{~cm} ; 1,06438 ; 1,03169$; 0,100207 . Já relativamente à largura das pontes, observamos, para os mesmos índices supracitados, valores equivalentes a $1,15 \mathrm{~cm}$; $1 \mathrm{~cm} ; 1,47915 ; 1,2162 ; 0,118128$, respectivamente.

Os resultados, quando tratados estatisticamente pelo método de Kruskal-Wallis, evidenciaram não existir relação significativa entre a altura ventricular e a largura da ponte $(\mathbf{H}=30,9213 \mathrm{e}$ valor crílico $=0,665401$ ) .

O Teste de Correlação de Person indica correlação positiva com tendência a nulidade quando confrontadas as alturas ventriculares com a vasculotopia $(r=0,0946)$ e correlações negativas com tendência à nulidade quando confrontadas as alturas ventriculares com as posiçôes $(r=-(0.1547)$, as posições com o número de pontes $(r=-(), 0680)$ e com a vasculotopia $(r=-0,0872)$, e a vasculotopia com as larguras $(r=-0,1603)$. Já correlações positivas de intensidade baixa são vistas em relação ao número e vasculotopia $(r=0,2037)$ e das posições com as larguras $(r=0,2352)$ e negativas com as alturas ventriculares e as larguras $(r=-0,2243)$, enquanto correlações de mediana intensidade são identificadas entre as alturas ventriculares e o número $(r=0,4126)$ e entre o número e as larguras $(r=-0,3803)$.

\section{DISCUSSÃO}

Um dos primeiros aspectos a serem considerados, a nosso ver, deve ser a alusão à nomenclatura anatômica utilizada na designação da estrutura em questão. Assim, necessário se faz afirmar que a denominação proposta nesta oportunidade, "ponte de miocárdio", está em concordância com as opiniões exaradas por Bezerra ${ }^{4}$ (1982), Bezerra et al. ${ }^{5}$ (1985), Amaral' (1989), Severino; Bombonato ${ }^{20}$ ( 1992 ).

Outra colocação que achamos pertinente fazer é o fato de que Berg ${ }^{2}$ (1963), ao estudar a mesma estrutura em corações de suínos, classificou-a como "anomalia". Afirmação esta discutível e da qual discordamos quer seja pelo conceito clássico de anomalia, que segundo Getty" (1981) diz respeito a "um grave desvio do padrão normal, acompanhado pela alteração ou depreciação da função", quer seja pelo nosso trabalho, em que a entidade em estudo estava presente em todas as peças.

Ainda, Berg apud Hadziselimovic et al. ${ }^{14}$ ( 1974) cita o aparecimento de "raras e pequenas" pontes de miocárdio para os bovinos, fato este muito diferente e portanto discordante do que ora encontramos para os bovinos azebuados, em que as mesmas aparecem com freqüência de $100 \%$, percentual este muito próximo do encontrado por Severino; Bombonato ${ }^{20}$ (1992) para os bovinos das 
raças Gir, Guzerá, Indubrasil e Nelore, em que aqueles autores relatam freqüência de $94 \%$ de animais portadores de pontes de miocárdio.

Em relação aos clássicos Tratados de Anatomia Veterinária, como por exemplo Bossi et al. ${ }^{\sharp}$ ( 1909); Bruni; Zimmerl" (1977); Ellenberger; Baum ${ }^{10}$ (1977); Gonzales y Garcia; Gonzales Alvarez'12 (1961); Montagné; Bourdellel6 (1917); Nickel et al. ${ }^{17}$ (1981); Schwarze; Schröder ${ }^{19}$ (1972) e Sisson; Grossman ${ }^{21}$ (1972), notamos que os autores, ao descreverem as artérias coronárias ou o próprio coração, não fazem menção à entidade por nós estudada, baseando seus relatos no comportamento e distribuição vascular ou na referência da estruturação das fibras musculares, no conjunto.

Hadziselimovic et al. ${ }^{14}$ (1974) descrevem que as artérias coronárias apresentam um "trajeto subepicardial e com formações musculares" sobre o ramo interventricular esquerdo em 3 corações de bovinos dos 20 da espécie por eles estudada. Citam, ainda, que as mesmas possuem de 2 a $4 \mathrm{~cm}$ de comprimento, estando localizadas com maior freqüência na posição dorsal ( $15 \%)$, determinando significativa diferença com os dados por nós obtidos, principalmente no que tange à freqüência. Os nossos achados são de certa forma muito semelhantes aos verificados por Severino; Bombonato ${ }^{20}$ (1992) e apenas em parte aos de Hadziselimovic et al. ${ }^{14}$ (1974), que encontram as pontes de miocárdio com maior frequência sobre o ramo interventricular paraconal $(39,6 \%)$, entretanto, num porcentual maior na posição média $(36,8 \%)$.

Em relação à frequência das pontes, vale ainda ressaltar os trabalhos de Bombonato et $a l .^{7}$ (1994) e Bertolini et $a l .^{3}$ ( 1994$)$ que, trabalhando com corações de suínos, indicam a sua presença em $36,36 \%$ e $32 \%$, respectivamente, sem diferenças estatísticas entre os sexos. Já Bombonato et al. ${ }^{\circ}(1991)$ assinalam frequência de $66,7 \%$ em gatos. Tais fatos, ainda que provenientes de animais de espécies e raças diferentes, distanciam-se em muito de nossos achados.

No que tange à largura das partes, Bombonato et $\mathrm{al}^{7}$ (1994) indicam para os suínos sem raça definida valores entre $0,2 \mathrm{~cm}$ e $1,9 \mathrm{~cm}$ e com média de $0,75 \mathrm{~cm}$, enquanto Bertolini et al. ${ }^{3}$ (1994) assinalam para animais desse mesmo grupo, mas de raças diferentes, dados variando entre $5,02 \mathrm{~mm}$ e $26,96 \mathrm{~mm}$, com média de $11,35 \mathrm{~mm}$ em corações cuja altura ventricular média foi de $93,17 \mathrm{~mm}$. Já Bombonato et al. ${ }^{6}$ (1991) descrevem medidas entre $1 \mathrm{~mm}$ e $6.7 \mathrm{~mm}$ com média de $3,6 \mathrm{~mm}$, em peças cuja altura média do ventrículo foi de $20,3 \mathrm{~mm}$ para os gatos. A comparação direta desses resultados com os agora obtidos permite-nos apenas ressaltar a necessidade de estudos comparativos não só entre as espécies de animais, mas dentro dessas variedades e raças, ficando a confrontação baseada nos dados estatísticos que em parte são coincidentes.

O número de pontes múltiplas encontradas nos corações de bovinos azebuados coincide com o dos relatos para os suínos (Bertolini et al. $^{3}, 1994$ ) e para os bovinos de raças determinadas (Severino; Bombonato ${ }^{21}, 1992$ ) e difere em parte do número de pontes encontrado para os suínos sem raça definida (Bombonato et al. ${ }^{7}, 1994$ ) e para os gatos (Bombonato et al. $\left.{ }^{6}, 1991\right)$, indicando claramente que, conforme os dados do tratamento estatístico empregado por aqueles autores, o número de pontes num mesmo coração independe da altura ventricular.

Nie; Vicent ${ }^{18}$ (1989) fazem uma colocação interessante quando se referem às pontes de miocárdio em corações de cães, propondo uma classificação balizada em suas larguras, denominando-as de pequenas $(<5 \mathrm{~mm})$, médias $(5$ a $10 \mathrm{~mm})$ e grandes $(>15 \mathrm{~mm}$ ). No entanto não se reportam à proporcionalidade ou correlação com altura ventricular ou cardíaca. Comparações relativas a esta classificação devem ser feitas com ressalvas, visto a diferença das alturas ventriculares dos animais estudados por aqueles e por nós.

Um outro ponto merecedor de consideração é que Nie; Vicent $^{18}$ (1989) fazem menção à presença de "pontes múltiplas" sobre um mesmo vaso. Este acontecimento é concordante com os nossos achados, em que identificamos $56,6 \%$ dos corações com pontes múltiplas, e difere, em parte, dos $40 \%$ encontrados para os corações de bovinos zebuínos descritos por Severino; Bombonato ${ }^{211}$ ( 1992).

No que concerne aos dados relativos à análise estatística, todos denotam não existirem relaçōes e correlações significativas de dependência das variáveis estudadas, mesmo naquelas de mediana intensidade; portanto não podendo ser concluído que quanto maior a altura do ventrículo, maior o número de pontes; nem quanto maior o número de pontes, menor a sua largura, dados estes em conformidade com os descritos por Severino; Bombonato ${ }^{20}$ (1992).

Nossos achados e os resultados descritos na literatura, especialmente aqueles oriundos dos animais domésticos, consolidam, a nosso ver, a necessidade da continuidade de estudos comparativos entre eles não somente pelas diferenças relativas à frequiência e posição, mas sobretudo nas correlações entre a largura das pontes e a altura ventricular.

\section{CONCLUSÕES}

Diante do que acabamos de expor, concluímos que:

1) As pontes de miocárdio nos bovinos azebuados estão presentes em todos os corações estudados;

2) Nestes animais, as pontes de miocárdio manifestamse em número de um a sete;

3) As pontes de miocárdio localizam-se, na maioria dos casos, sobre colaterais da artéria coronária esquerda, podendo ocorrer ocasionalmente sobre ramos da artéria coronária direita;

4) As pontes de miocárdio encontradas nos corações examinados localizam-se mais freqüentemente nas regiões média e apical;

5) As pontes de miocárdio nos corações de bovinos azebuados estão localizadas, mais freqüentemente, no ramo interventricular paraconal;

6) As múltiplas pontes de miocárdio sobre um único vaso foram observadas em número de 2 a 4 , com maior freqüência de 4 e sobre o ramo interventricular paraconal. 


\section{SUMMARY}

Thirty hearts of males cattle from 30 to 50 months old were studied, in order to observe the frequency, width and localization of the myocardial bridges on the coronary arteries. Hearts had their coronary arteries injected with colored solution of Neoprene latex 450 , what was followed by dissection. Myocardial bridges were observed in $100 \%$ of the hearts. They were multiple in $56,6 \%$ of the cases. The width of those bridges ranged from 0.3 to $6.2 \mathrm{~cm}$ with an avarege of $1.62 \mathrm{~cm}$. Bridges were most frequently found in the medium portion of the ventriculus. The most common site of myocardial bridges was the paraconal interventricular branch.

UNITERMS: Bovines; Myocardial bridges; Heart; Arteries.

\section{REFERÊNCIAS BIBLIOGRÁFICAS}

1- AMARAl R.C. Pontes de miocárdio em cães. São Paulo, 1989.96 p. Tese (Doutorado). Faculdade de Medicina Veterinária e Zootecnia, Universidado de São Paulo.

2 - BERG, R. Über das Auftrelen von Myocardbrücken den Koronargefäben bein Schwein (Sus scrofa domesticus). Anatomischer Anzeiger, v. 112, n. 1. p. 25 $-31,1963$.

3 - BERTOLINI. S.M.M.G.; PRATLS, N.E.V.B.; MIRANDA NETO, M.H. Estudo macrosép ptco e estatístico das pontes de miocárdio sobre as artérias coronárias de suínos. Revista UNIMAR, v. 16, n. 2, p. 383-93, 1994.

4 - BEZERRA. A. J. C Contribuição para o conhecimento das pontes de miocárdio. São Paulo, 1982. 169 p. Dissertação (Mestrado). Escola Paulista de Medicina

5 - BEZERRA, A. J. C. : DIDIO, A. S.: DIDIO, I. J. A. Bridges of myocardium over branches of the coronary arteries in Camelus dromedarius. Archivio Italiano di Anatomia e di Embriologia, v. 90, n. 4, p. 267-74, 1985

6- BOMBONATO, P.P.: MARIANA, A.N.B.: AMARAL, R.C. SANTOS, A.L.Q: SILVA. FO.C. SEVERINO, R.S. Pontes de miocárdio em gatos. Revista Científica do Centro de Ciências Biomédicas da Universidade Federal de Uberlândia. v. 7, n. 1. p. 49-57. 1991

7 - BOMBONATO, P.P.; DOMINGOS, C.A; MARIANA, A.N.B.; SILVA, F.O.C.; INTELIZANO. W. Ocorrência de pontes de miocárdio em suínos. Brazilian Journal of Veterinary Research and Animal Science, v. 31, n. 2, p. 107-11. 1994.

8 - BOSSI, V.; CARADONNA, G. B.: SPAMPANI, G.: VARALD, I.: ZIMMERI. U. Trattato di anatomia veterinaria. Milano, Francesco Vallardi, 1909, v.2. p. $3-44$

9 - BRUN1, A. C.; ZIMMERL. U. Anatomia degli aninali domestici 2.ed. Milano, Francesco Vallardi, 1977. v. 2 , p.290-304
I0 - ELLJENBERGER, W: BAUM. II. Handbuch der vergleichenden anatomie der haustiere. 18.ed. Berlin, Springer, 1977, p. 610-29.

11 - GITTT, R. Sisson / Grossman anatomia dos animais domésticos 5. ed. Rio de Janciro, Interamericana, 1981. V. I, p. 90()-2.

12 - GONZALES Y GARCIA, J.; GONZALES ALVARLZ, R. Anatomia comparada de los animales domesticos. 7. ed. Madrid, Canales, 1961 p. $59-606$.

13 - HABERMEHL, K. H. Die Blutgefasversorgung des Katzenherzens. Zentralblatt fïir Veterinarmedizin, v. 6, p. 655-80, 1959

14 - HAIDZISELIMOVIC, H.: SECEROV. [): GMAZ-NIKULIN, E. Comparative analomical investigation on coronary arteries in wild and domeslic animals. Acta Anatomica, v. 90, n. I, p. 16-35, 1974

15 - HEGAZI, II. Die Blutgefassversorgung der Herzens von Rind, Schaf und Ziege. Giessen, 1958. 36 p. Thesis (Doktorgrades). Sachbereish Veterinarmedizill und Tiervuzht der Jestus Lichig Univerdität.

16 - MONTAGNÉ, L.; BOURDELLE, F. Anatomie régionale des animaux domestiques. Paris, J - B Baillière, 1917. v. 2 , p. 24()-8.

17 - NICKEL, R.; SCHUMMER. A.: SEIFERLE. E. The anatomy of the domestic animals. Berlin, Paul Parey, 1981. v. 3, p. 54-62

18 - NIE. C. J.; VICENT, J. G. Myocardial bridges on the coronary arteries in animals. Acta Anatomica. v. 18. p. 45-51. 1989

19 - SCHWARZE, E; SCHRODER, L. Compendio de anatomia veterinária Zaragoza, Acribia, 1972. v. 3, p. 14-27.

20 - SEVERINO, S.: BOMBONATO, P. P. Ocorrência de pontes de miocírdio em hovinos das raças Gir, Guzerá. Indubrasil e Nelore. Brazilian Journal of Veterinary Research and Animal Science, v. 29, n. 1, p. 15-30, 1992.

21 - SISSON, S.; GROSMAN, J. Anatomia de los animales domesticos. 4 ed Barcelona, Salvat, 1972. p. 696-8. 